06,19

\title{
Электрокалорический эффект в триглицинсульфате в равновесных и неравновесных термодинамических условиях
}

\author{
(C) В.С. Бондарев ${ }^{1,2}$, Е.А. Михалёва ${ }^{1}$, И.Н. Флёров ${ }^{1,2}$, М.В. Горев ${ }^{1,2}$ \\ ${ }^{1}$ Институт фризики им. Л.В. Киренского, ФИЦ КНЦ СО РАН, \\ Красноярск, Россия \\ ${ }^{2}$ Институт инженерной фризики и радиоэлектроники Сибирского федерального университета, \\ Красноярск, Россия \\ E-mail: vbondarev@yandex.ru
}

(Поступила в Редакцию 22 ноября 2016 г.)

\begin{abstract}
Выполнены прямые и косвенные измерения интенсивного электрокалорического эффекта в сегнетоэлектрическом кристалле триглицинсульфата в равновесных и неравновесных термодинамических условиях, реализованных в адиабатическом калориметре. Исследовано влияние параметров (частоты, профиля, напряженности) электрического поля на величину эффекта и степень его обратимости. В квазиизотермических условиях установлено различие величин изменения температуры при приложении и снятии постоянного поля. Периодическое электрическое поле низкой частоты вызывает появление градиента температур вдоль электрокалорического элемента и теплового потока от его „свободного“ торца к термостатированному основанию. Значительное превышение скорости выключения над скоростью включения поля приводит к заметному увеличению эффекта охлаждения.
\end{abstract}

Исследование выполнено при финансовой поддержке Российского фонда фундаментальных исследований, Правительства Красноярского края, Красноярского краевого фонда поддержки научной и научно-технической деятельности в рамках научного проекта № 16-42-240428 р_а.

DOI: 10.21883/FTT.2017.06.44482.421

\section{1. Введение}

Одно из перспективных направлений поиска новых методов охлаждения и разработки эффективных рефрижераторных устройств, активно развиваемых в последнее десятилетие научным и инженерным сообществами, связано с исследованиями калорических эффектов (СЕ) разной физической природы в твердых телах [1-8]. В основе такого рода явлений лежит принцип обратимого изменения температуры $\Delta T_{\mathrm{AD}}$ или энтропии $\Delta S_{\mathrm{CE}}$ твердого тела за счет воздействия на него внешнего поля (электрического, магнитного, механических напряжений) соответственно в адиабатных или изотермических условиях $[1,4,8]$. Внешнее поле приводит к изменению температурной зависимости соответствующего параметра порядка (поляризации, намагниченности, деформации), что, в свою очередь, вызывает охлаждение/нагревание твердотельного элемента (хладагента). А периодическое и согласованное изменение напряженности поля, прикладываемого к рабочему элементу, и условий термодинамического сопряжения хладагента с окружающей средой позволяет осуществить замкнутый холодильный цикл. Так как наиболее существенные изменения параметра порядка с температурой и его восприимчивости к изменению поля имеют место в области фазовых переходов, то и СЕ достигают наибольших значений в этом интервале температур. Именно этим объясняется повышенный интерес исследователей к материалам, испытывающим фазовые переходы электрической, магнитной или упругой природы.
Благодаря высокой плотности твердых тел охлаждающие устройства на их основе могут быть в значительной мере более компактными по сравнению с традиционными парожидкостными холодильными установками. Таким образом, использование твердотельных хладагентов в рефрижераторных устройствах является не только целесообразным, но и предпочтительным.

$\mathrm{C}$ точки зрения технической реализации электрокалорический эффект (ЕСЕ), по сравнению с магнетокалорическим (МСЕ) и барокалорическим (ВСЕ) эффектами, является наиболее простым, так как достаточно приложить напряжение к электродам, нанесенным на противоположные грани кристаллического или керамического элемента. И это, в частности, является одной из причин роста в последние годы количества исследований и соответствующих публикаций, посвященных $\operatorname{ECE}[3,4,9,10]$. Одной из основных целей такого рода работ является поиск путей создания новых и модернизации известных материалов, позволяющих получить большие значения абсолютных величин интенсивного $\Delta T_{\mathrm{AD}}$ и экстенсивного $\Delta S_{\mathrm{ECE}}$ эффектов. Однако при этом неизбежной оказывается необходимость в больших напряженностях электрического поля $E$, нередко приводящих к электрическому пробою объемных электрокалорических (ЕС) элементов [11]. Последнюю проблему пытаются решить путем использования пленочных сегнетоэлектрических элементов, в которых большие величины $E$ могут быть реализованы при невысоких напряжениях [12,13]. И это позволило получить довольно обнадеживающие результаты по увеличению интенсивного ЕСЕ, практически 
приближенные к лучшим, реализованным в ферромагнетиках вследствие MCE $[2,8]$. Однако в силу незначительного объема (массы) пленочных элементов, экстенсивный ЕСЕ оказывается настолько мал, что говорить о перспективности их применения рано.

С учетом приведенных выше проблем, характерных для сегнетоэлектрических калорических элементов, исследуемых и планируемых к применению в равновесных тепловых и электрических условиях, весьма перспективным выглядит направление, связанное с гипотетической возможностью значительного усиления ЕСЕ в условиях периодически меняющегося поля, приложенного к твердым телам, находящимся в неравновесных тепловых условиях. Теоретически такая задача была недавно решена для сегнетоэлектриков, обладающих высокими значениями диэлектрической восприимчивости $\chi$ и значительной нелинейностью производной $\partial \chi / \partial T$ [14-16]. В результате воздействия периодического поля $E$ на образец, один край которого термостатирован, возникает градиент температуры, появляется динамическая поляризация и тепловой поток. В параэлектрической фазе количество поглощенного тепла больше выделенного, что и является причиной охлаждения элемента в неравновесных тепловых условиях.

Недавно нами было выполнено экспериментальное исследование ЕСЕ под воздействием переменного $E$ в сегнетоэлектрическом элементе, изготовленном в виде четырехгранной прямоугольной призмы из кристалла триглицинсульфата (TGS) [17]. В качестве наиболее важного методического момента рассматривалась необходимость поддержания постоянства температуры основания призмы. Измерения проводились в условиях среднего вакуума $\left(10^{-2} \mathrm{~mm} \mathrm{Hg}\right)$ и без дополнительных мер по исключению теплообмена образца с окружающей средой. В результате наблюдалось охлаждение калорического элемента, зависящее, как и предполагалось в [14-16], от частоты $E$. Однако разница температур между термостатированным основанием и „свободным“ торцом ЕС-элемента оказалась намного меньше, чем следовало из расчетов, выполненных с учетом условий, реализованных в экспериментах [17]. Предполагалось, что причина небольшого эффекта охлаждения элемента TGS связана с теплообменом с окружающей средой, с плохим тепловым контактом между датчиком температуры и ЕС-элементом и с обнаруженной проводимостью исследуемого образца. Целый ряд перечисленных выше причин можно устранить, или уменьшить их влияние, выполняя измерения в адиабатических условиях. С этой точки зрения наиболее подходящим методом исследования интенсивного ЕСЕ является адиабатический калориметр. Этот метод обладает высокой чувствительностью к незначительным изменениям температуры $\Delta T_{\mathrm{AD}}$ и позволяет также определить и экстенсивный $\Delta S_{\mathrm{ECE}}$ эффект из измерений и анализа теплоемкости в зависимости от напряженности электрического поля.

В настоящей работе продолжены исследования ЕСЕ в сегнетоэлектрическом кристалле TGS как в равновесных, так и неравновесных термодинамических условиях.
Bсе эксперименты выполнены в адиабатическом калориметре с системой тепловых экранов, позволяющих в наибольшей мере исключить теплообмен образца с окружающей средой [18]. Выполнены прямые и непрямые исследования ЕСЕ при варьировании параметров электрического поля.

\section{2. Образцы и методы исследований}

Исследования в настоящей работе были выполнены на образцах, приготовленных из массивного монокристалла TGS, выращенного в параэлектрической фазе из водного раствора в максимально равновесных тепловых условиях и использовавшегося нами ранее для исследований ECE в [17]. На порошковых рентгенограммах TGS не обнаружено рефлексов, указывающих на присутствие в кристалле посторонних фаз и примесей. Определенные в [17] температура фазового перехода $P 2_{1} / m \leftrightarrow P 2_{1}$ $T_{\mathrm{C}}=322.37 \mathrm{~K}$, максимальная диэлектрическая проницаемость $\varepsilon_{b}^{\max }=3 \cdot 10^{4}$, а также величина поляризации $\left(P=2.5 \mu \mathrm{C} \cdot \mathrm{cm}^{-2}\right.$ при $\left.290 \mathrm{~K}\right)$ и характер ее поведения удовлетворительно согласуются с результатами предыдущих исследований $[19,20]$.

Специфика СE, в том числе ECE, такова, что прямым путем может быть измерен только интенсивный эффект $\Delta T_{\mathrm{AD}}$. Для определения экстенсивного эффекта $\Delta S_{\mathrm{CE}}$ используют различные косвенные методы. В соответствии с анализом и сравнительной оценкой разнообразных методов исследования ЕСЕ, выполненных недавно в [21], оба подхода, прямой и непрямой, имеют право на использование, но, безусловно, метод непосредственного измерения $\Delta T_{\mathrm{AD}}$ более предпочтителен.

В соответствии с сутью интенсивного ЕСЕ его реальная величина должна определяться при включении/выключении электрического поля в условиях постоянства энтропии исследуемого образца. С другой стороны, один из путей получения информации об экстенсивном ЕСЕ заключается в анализе данных о температурных зависимостях теплоемкости в условиях $E=0$ и $E \neq 0$. Для совокупного решения такого рода задач наиболее оптимальным является метод адиабатического калориметра. К тому же, как будет показано далее, в таком калориметре возможно проведение экспериментов по определению интенсивного ЕСЕ в неравновесных условиях и при варьируемой частоте электрического поля.

Использованная в работе калориметрическая установка аналогична описанной в [18]. Здесь мы представляем только особенности монтажа образца и конструкции измерительной ячейки (рис. 1).

Весь комплекс исследований проведен на одном образце TGS в виде прямоугольного параллелепипеда с размерами $(10 \times 6 \times 1) \mathrm{mm}^{3}$ (1) (рис. 1,a). На наибольшие по площади грани ЕС-элемента, перпендикулярные сегнетоэлектрической оси $b$, методом вакуумного напыления нанесены золотые электроды (2), к 


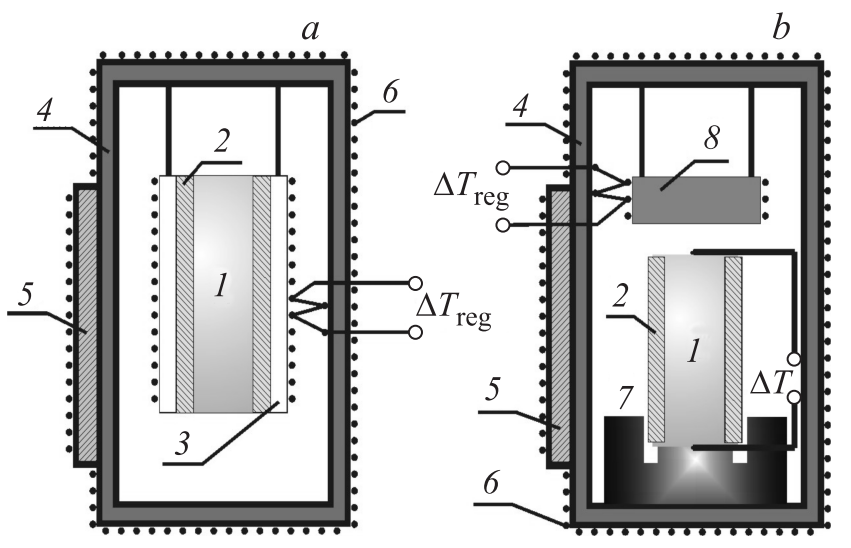

Рис. 1. Схема монтажа образца для исследования теплоемкости и ЕСЕ в адиабатических условиях $(a)$ и влияния на ЕСЕ квазиизотермических процессов наложения/снятия постоянного и периодического электрических полей $(b)$.

которым прикладывалось напряжение, создающее внутри образца электрическое поле $E$. Для предотвращения электрического пробоя поверх электродов наклеивались тонкие $(\sim 0.2 \mathrm{~mm})$ слюдяные пластинки (3). Подготовленный образец был упакован в полированную алюминиевую фольгу с наклеенным нагревателем. Надежный тепловой контакт между элементами системы „образец + фурнитура“( (слюда + фольга с нагревателем) обеспечивался с помощью вакуумной смазки.

Для измерений ECE и теплоемкости в равновесных условиях система подвешивалась на тонких нитях $(d \leq 0.1 \mathrm{~mm})$ внутри адиабатического экрана калориметра (4) (рис. 1,a). Температура образца контролировалась с помощью прецизионного платинового термометра сопротивления (5), смонтированного на адиабатическом экране. Контроль и регулирование разности температур $\Delta T_{\text {reg }}$ между адиабатическим экраном и образцом осуществлялись с помощью батареи медь-константановых термопар, сигнал от которых поступал в цепь прецизионного усилителя мощности, связанного с нагревателем адиабатического экрана (6). Минимизация радиационного и конвективного теплообменов осуществлялась дополнительными экранами и помещением всей измерительной системы в герметичную оболочку с высоким вакуумом $\sim 10^{-5} \mathrm{~mm} \mathrm{Hg}[18]$.

Исследования интенсивного ЕСЕ в неравновесных тепловых условиях „релаксационным“ методом были выполнены следующим образом (рис. 1,b). Основание образца TGS с электродами (1) помещалось в медный держатель (7), который в свою очередь прикреплялся к адиабатическому экрану (4). Над образцом к крышке экрана подвешивался медный диск (8) $(d=7.94 \mathrm{~mm}$, $h=1.89 \mathrm{~mm})$, упакованный в полированную алюминиевую фольгу с нагревателем и выполняющий роль дополнительного теплового экрана. Для обеспечения надежного теплового контакта между всеми элементами системы использовалась вакуумная смазка. Система ав- томатического регулирования позволяла поддерживать необходимую разность температур между медным диском и адиабатическим экраном с точностью не хуже $10^{-4} \mathrm{~K}$. Таким образом, осуществлялось постоянство температуры адиабатического экрана и тем самым реализовались изотермические условия для основания образца TGS $\left(T_{\text {bot }}=\right.$ const). Разница температур между верхним „свободным“ и нижним термостатированным торцами образца $T_{\text {top }}-T_{\text {bot }}$, возникающая за счет ЕCE при включении/выключении $E$, фиксировалась с помощью термопары медь-константан. Чувствительность датчиков температуры и стабильность измерений составляли соответственно $10^{-4}$ и $5 \cdot 10^{-4} \mathrm{~K}$.

\section{3. Результаты и обсуждение}

3.1. Исследование влияния электрического поля на теплоемкость. На первом этапе были выполнены исследования влияния электрического поля на теплоемкость $C(T, E)$ образца TGS традиционным методом адиабатического калориметра. Измерения проведены в интервале температур 270-335 К методами дискретных и непрерывных нагревов со скоростями $d T / d t=2.3 \mathrm{~K} \cdot \mathrm{h}^{-1}$ и $d T / d t=0.2 \mathrm{~K} \cdot \mathrm{min}^{-1}$. Теплоемкость фурнитуры, измеренная в отдельном эксперименте, составила $\sim 50 \%$ от общей теплоемкости системы „образец + фурнитура“.

Так как мы будем анализировать ЕСЕ вблизи температуры фазового перехода, то на рис. 2, $a$ представлены зависимости $C(T, E)$ в узкой области температур, измеренные при напряженностях электрического поля в интервале $0-2.8 \mathrm{kV} \cdot \mathrm{cm}^{-1}$. При $E=0$ аномалия теплоемкости, соответствующая фазовому переходу сегнетоэлектрик-параэлектрик, наблюдалась при $T_{C}=322.25 \pm 0.05 \mathrm{~K}$, что хорошо согласуется с полученными ранее данными $[17,19]$. Характер поведения теплоемкости под полем соответствует наблюдавшимся ранее [19]: уменьшению максимума и размытию скачка теплоемкости.

Для получения информации об аномальной энтропии $\Delta S_{\text {an }}(T, E)=\int\left[\Delta C_{\text {an }}(T, E) / T\right] d T$, связанной с фазовым переходом, необходимо было выделить решеточную $C_{\mathrm{L}}(T)$, не зависящую от $E$, и аномальную $\Delta C_{\text {an }}(T, E)$ теплоемкости. Решеточный вклад определялся аппроксимацией полной теплоемкости вдали от температуры фазового перехода комбинацией функций Дебая и Эйнштейна. Поведение аномальной энтропии в зависимости от температуры и напряженности электрического поля показано на рис. $2, b$.

Температурные зависимости экстенсивного ЕСЕ для различных напряженностей электрического поля, определенного как разность энтропий при $E \neq 0$ и $E=0$

$$
\begin{aligned}
\Delta S_{\mathrm{ECE}}(T, E) & =S(T, E)-S(T, 0) \\
& =\Delta S_{\mathrm{an}}(T, E)-\Delta S_{\mathrm{an}}(T, 0),
\end{aligned}
$$

представлены на рис. 2, c. 

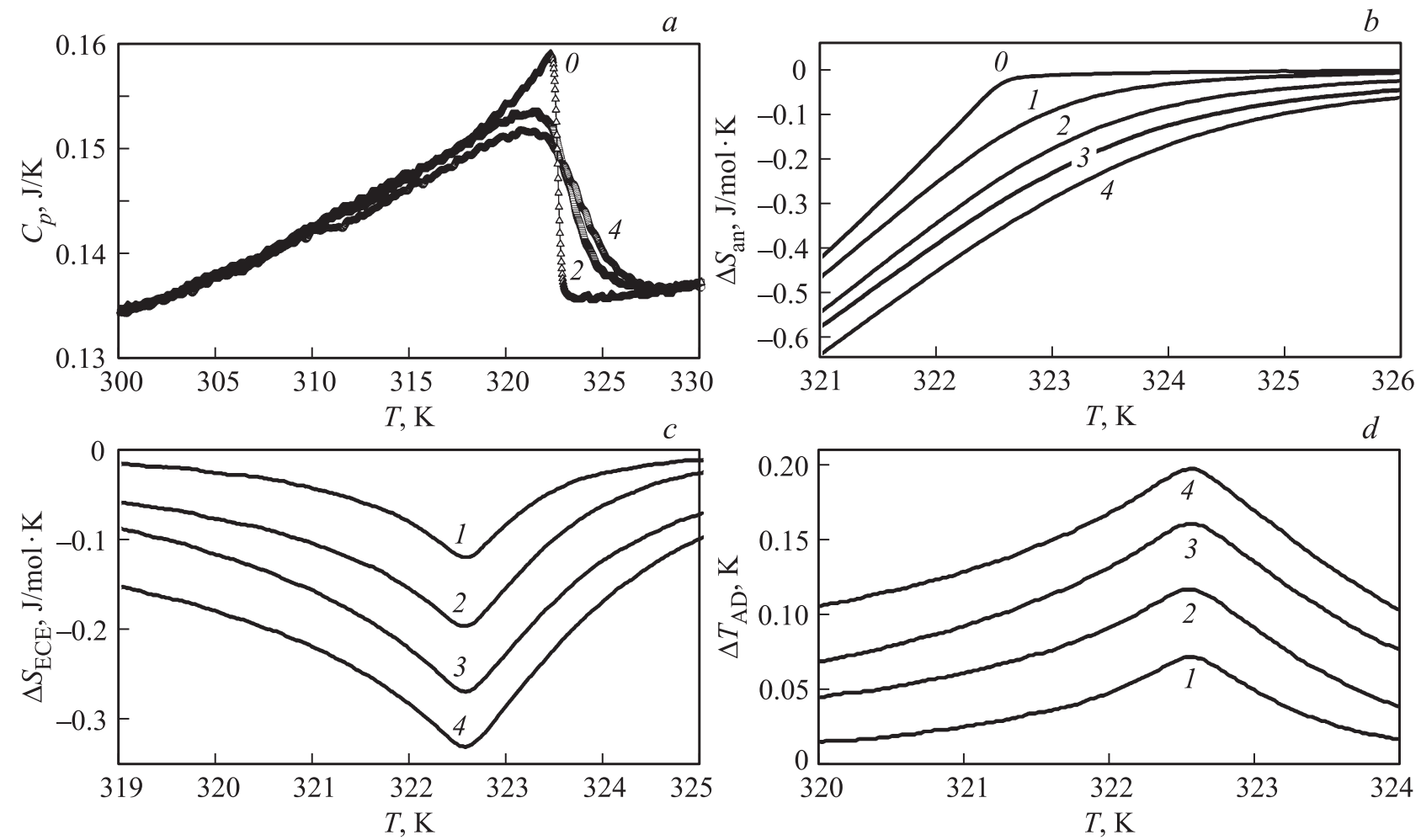

Рис. 2. Температурные зависимости теплоемкости системы „образец + фурнитура“ при постоянных полях (a), аномальной энтропии $\Delta S_{\mathrm{an}}(T, E)$ TGS $(b)$, экстенсивного $\Delta S_{\mathrm{ECE}}(c)$ и интенсивного $\Delta T_{\mathrm{AD}}(d)$ ЕСЕ. Номера кривых соответствуют напряженностям $E\left(\mathrm{kV} \cdot \mathrm{cm}^{-1}\right): 0-0.0 ; 1-0.7 ; 2-1.4 ; 3-2.1 ; 4-2.8$.

Интенсивный $\mathrm{ECE} \Delta T_{\mathrm{AD}}(T, E)$ был определен из данных о полной энтропии образца $S(T, E)$, исходя из условия

$$
S(T, E)=S\left(T+\Delta T_{\mathrm{AD}}, 0\right) .
$$

Максимальные величины $\Delta S_{\mathrm{ECE}}^{\max }=-0.329 \mathrm{~J} \cdot(\mathrm{mol} \cdot \mathrm{K})^{-1}$ и $\Delta T_{\mathrm{AD}}^{\max }=0.198 \mathrm{~K}$ при $E=2.8 \mathrm{kV} \cdot \mathrm{cm}^{-1}$ наблюдались при $T_{\mathrm{ECE}}^{\max }=322.54 \pm 0.05 \mathrm{~K}$, то есть выше температуры фазового перехода $T_{C}$ при $E=0$. Отличие этих температур вызвано тем, что $T_{C}$ соответствует температуре максимума аномальной теплоемкости, связанной с температурной производной квадрата поляризации, $\Delta C_{\text {an }}^{\max } \sim\left(\partial \Delta S_{\text {an }} / \partial T\right)^{\max } \sim\left(\partial P^{2} / \partial T\right)^{\max }[22]$, a $T_{\mathrm{ECE}}^{\max }$ определяется соотношением $\Delta S_{\mathrm{ECE}}^{\max } \sim \Delta T_{\mathrm{AD}}^{\max }$ $\sim(\partial P / \partial T)^{\max }[4]$.

\section{2. Прямые измерения интенсивного} ECE в адиабатических условия. Классический эксперимент по прямому определению интенсивного ЕСЕ в адиабатических условиях в соответствии со схемой, представленной на рис. $1, a$, состоял из последовательных этапов (рис. 3, $a$ ). Система „образец + фурнитура“ нагревалась/охлаждалась до температуры, при которой в дальнейшем выполнялись исследования, и задавался линейный дрейф температуры с оптимальной скоростью в пределах $d T / d t \approx \pm(1-5) \cdot 10^{-4} \mathrm{~K} \cdot \mathrm{min}^{-1}$. Затем к электродам образца прикладывался импульс электрического напряжения продолжительностью 5-10 min, что приводило за счет ЕСЕ к резкому возрастанию температуры системы на величину $\Delta T_{\exp }^{\mathrm{ON}}$, которая меньше $\Delta T_{\mathrm{AD}}$ из-за потерь тепла на нагревание „пассивных“ элементов системы слюды, нагревателя и смазки. После выключения поля температура образца резко понижалась на величину $\Delta T_{\exp }^{\mathrm{OFF}}$. Затем процесс повторялся при другой напряженности электрического поля (рис. 3,a). Погрешность определения величины $\Delta T_{\exp }$ не превышала $\pm 2 \cdot 10^{-4} \mathrm{~K}$.

Незначительное изменение температуры в течение каждого эксперимента (рис. 3, $a$ ) за счет весьма малого ее дрейфа $d T / d t \approx 3.3 \cdot 10^{-4} \mathrm{~K} \cdot \mathrm{min}^{-1}$ при $E=0$ обеспечивало адиабатические условия. При $E \neq 0$ скорость изменения температуры системы после переходного периода не должна отличаться от $(d T / d t)_{E=0}$. Однако в эксперименте она оказалась значительно большей и достигала $d T / d t \approx 6.8 \cdot 10^{-4} \mathrm{~K} \cdot \mathrm{min}^{-1}$ при $E=2.8 \mathrm{kV} \cdot \mathrm{cm}^{-1}$. Это обстоятельство свидетельствует о наличии проводимости в исследуемом кристалле TGS вблизи фазового перехода, что приводит при $E \neq 0$ к выделению джоулева тепла на сопротивлении образца. Заметная проводимость кристалла TGS явилась причиной того, что после снятия электрического поля температура образца не возвращалась на ожидаемый уровень, соответствующий экстраполяции предыдущего хода температуры (рис. $3, a$ ). Величина $\Delta T_{\exp }$ определялась как разность температур, полученных линейной экстраполяцией зависимостей $T(t)$ к моменту включения или выключения поля. 

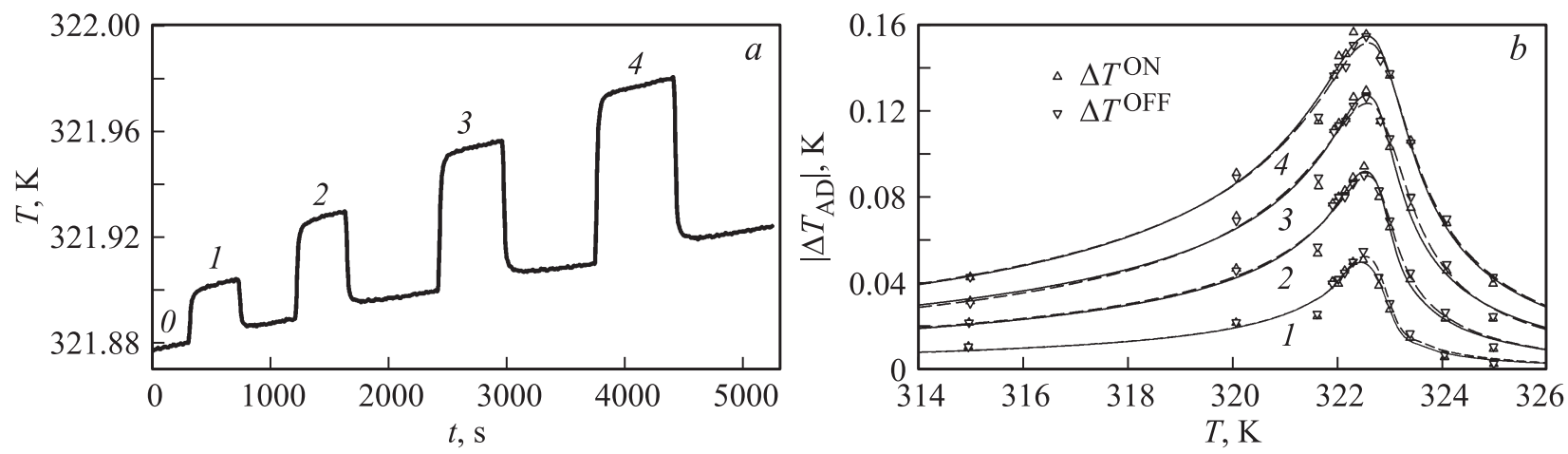

Рис. 3. Зависимость температуры системы „образец + фурнитура“ от напряженности электрического поля $\left(\mathrm{kV} \cdot \mathrm{cm}^{-1}\right): 0.0(0)$; $0.7(1) ; 1.4(2) ; 2.1(3) ; 2.8(4)(a)$. Температурные зависимости интенсивного ЕСЕ, определенные из (3), для режимов включения $\left(\Delta T_{\mathrm{AD}}^{\mathrm{ON}}\right)$ и выключения $\left(\Delta T_{\mathrm{AD}}^{\mathrm{OFF}}\right)$ электрического поля $(b)$.

Для определения действительной величины $\Delta T_{\mathrm{AD}}$ необходима корректировка экспериментально опреде-

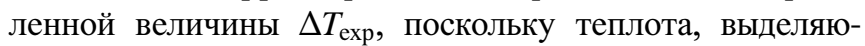
щаяся за счет ЕСЕ, расходуется на изменение температуры не только образца, но всей системы „образец + фурнитура“ в целом. Связь между величинами $\Delta T_{\exp }$ и $\Delta T_{\mathrm{AD}}$ устанавливается соотношением

$$
\Delta T_{\mathrm{AD}}=\Delta T_{\exp }\left(1+\frac{C_{f}}{C_{\mathrm{smp}}}\right)
$$

где $C_{f}-$ теплоемкость фурнитуры, определенная в отдельном эксперименте, $C_{\text {smp }}$ - теплоемкость образца.

На рис. $3, b$ представлены температурные зависимости интенсивного ЕСЕ, скорректированные в соответствии с (3), при включении $\Delta T_{\mathrm{AD}}^{\mathrm{ON}}$ и выключении $\Delta T_{\mathrm{AD}}^{\mathrm{OFF}}$ электрического поля. При $E=2.8 \mathrm{kV} \cdot \mathrm{cm}^{-1}$ интенсивный $\mathrm{ECE}$ достигает максимума $\left|\Delta T_{\mathrm{AD}}\right|^{\max }=0.154 \mathrm{~K}$ при температуре $322.53 \pm 0.05 \mathrm{~K}$, совпадающей $\mathrm{c}$ $T_{\mathrm{ECE}}^{\mathrm{max}}=322.54 \mathrm{~K}$, определенной из анализа зависимостей $S(T, E)$. Однако величины $\left|\Delta T_{\mathrm{AD}}\right|^{\max }$, полученные при прямых измерениях, оказались меньше вычисленных при тех же напряженностях $E$ (рис. $2, d$ ) в пределах $20 \%$. Такое расхождение для величин $\Delta T_{\mathrm{AD}}$, определенных прямыми и косвенными методами, встречается нередко для СЕ разной природы $[21,23]$.

Изменение температуры после включения электрического поля различной напряженности $\Delta T_{\mathrm{AD}}^{\mathrm{ON}}$ в пределах нескольких процентов совпадало с величиной $\Delta T_{\mathrm{AD}}^{\mathrm{OFF}}$, наблюдавшейся в процессе выключения поля. Например, при $E=2.8 \mathrm{kV} \cdot \mathrm{cm}^{-1}$ и $T=323.0 \mathrm{~K}$ эти величины составили соответственно $+0.136 \mathrm{~K}$ и $-0.137 \mathrm{~K}$. Полученные в результате многократных измерений данные свидетельствуют о высокой степени воспроизводимости и обратимости интенсивного ECE в кристалле TGS в адиабатических условиях и вполне удовлетворительно согласуются с данными, полученными ранее только для $\Delta T_{\mathrm{AD}}^{\mathrm{ON}}[24]$.

Следует заметить, что в наших экспериментах включение и выключение $E$ происходило при разных, хотя и мало отличающихся, температурах, а именно, при $T$ и $T+\Delta T_{\mathrm{AD}}$ (рис. 3, $a$ ). Проведены также измерения ЕСЕ, когда включение и выключение поля $E$ можно было выполнить при одной и той же температуре (рис. 4, $a):(T, E=0) \rightarrow\left(T+\Delta T_{\mathrm{AD}}^{\mathrm{ON}}, E \neq 0\right)$ и $(T, E \neq 0) \rightarrow\left(T-\Delta T_{\mathrm{AD}}^{\mathrm{OF}}, E=0\right)$. Для этого задавалась отрицательная скорость изменения температуры системы „образец + фурнитура“, величина которой $d T / d t=-2.5 \cdot 10^{-3} \mathrm{~K} / \mathrm{min}$ по модулю на порядок превышала скорость в экспериментах с $d T / d t>0$, представленных на рис. 3, $a$. Довольно большая скорость охлаждения системы в условиях $E=0$ была необходима, чтобы в процессе $E \neq 0$ температура, при которой было включено поле, достигалась в течение $10-30 \mathrm{~min}$. Некоторые результаты такого рода экспериментов представлены на рис. $4, b$.

Обнаружено различие величин $\Delta T_{\mathrm{AD}}^{\mathrm{ON}}$ и $\Delta T_{\mathrm{AD}}^{\mathrm{OFF}}$, которое превышает ошибку измерений и имеет разные знаки в сегнетоэлектрической $\left|\Delta T_{\mathrm{AD}}\right|^{\mathrm{ON}}(T, E)>\left|\Delta T_{\mathrm{AD}}\right|^{\mathrm{OFF}}(T, E)$ и параэлектрической $\left|\Delta T_{\mathrm{AD}}\right|^{\mathrm{ON}}(T, E)<\left|\Delta T_{\mathrm{AD}}\right|^{\mathrm{OFF}}(T, E)$ фазах. Так, например, поле $E=1.4 \mathrm{kV} \cdot \mathrm{cm}^{-1}$ приводит к следующим эффектам: при $T=321 \mathrm{~K}<T_{C} \Delta T_{\mathrm{AD}}^{\mathrm{ON}}=$ $=0.060 \mathrm{~K}$ и $\Delta T_{\mathrm{AD}}^{\mathrm{OFF}}=-0.056 \mathrm{~K}$, а при $T=323.5 \mathrm{~K}>T_{C}$ $\Delta T_{\mathrm{AD}}^{\mathrm{ON}}=0.046 \mathrm{~K}$ и $\Delta T_{\mathrm{AD}}^{\mathrm{OFF}}=-0.050 \mathrm{~K}$.

3.3. Исследования интенсивного ЕCE „релаксационным“ методом в квазиизотермических условиях. Исследования ЕСЕ „релаксационным“ методом были выполнены на образце TGS, смонтированном в соответствии со схемой, представленной на рис. $1, b$. Такие условия являются квазиизотермическими, так как в процессе измерений постоянной поддерживалась только температура основания образца $T_{\mathrm{bot}}$ В результате быстрого (адиабатического) включения/выключения электрического поля температура верхнего „свободного“ торца образца быстро росла/убывала в результате ЕСЕ и затем при $E=$ const довольно медленно релаксировала до значения, соответствующего температуре $T_{\text {bot }}$ (рис. $5, a$ ).

Возникающий градиент температур на образце приводил к появлению теплообмена между нижним (термо- 

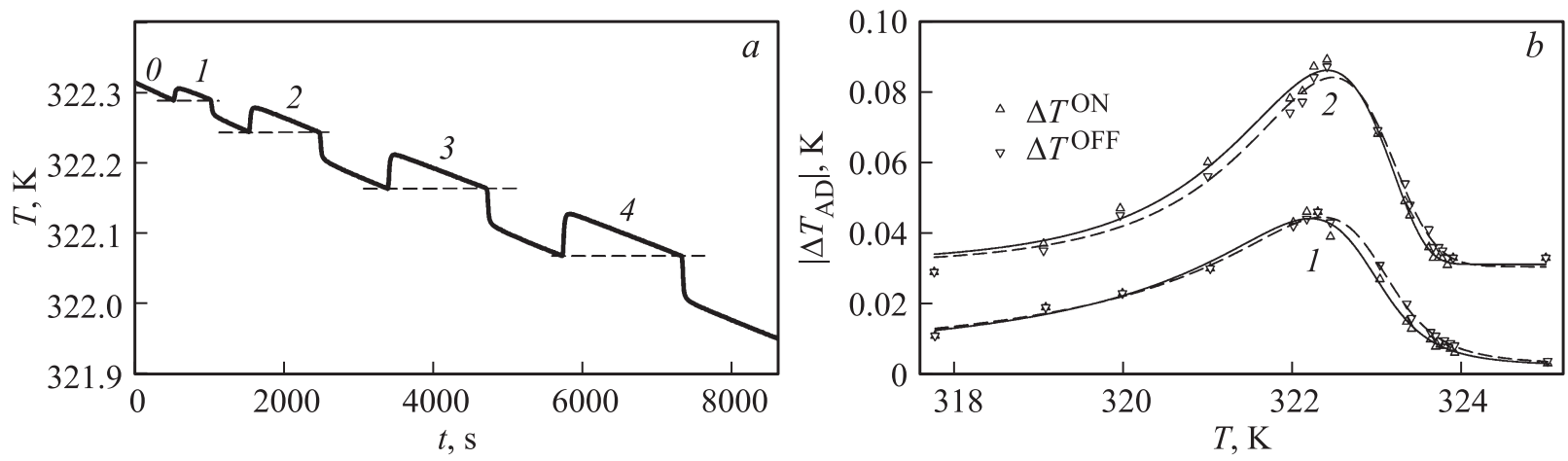

Рис. 4. ECE в кристалле TGS в квазиизотермических тепловых условиях; результаты измерений $\Delta T_{\text {exp }}$ с поэтапным увеличением $E(a)$; температурные зависимости $\Delta T_{\mathrm{AD}}$ вблизи фазового перехода. Номера кривых соответствуют напряженностям $E(\mathrm{kV}$. $\left.\mathrm{cm}^{-1}\right): 0-0.0 ; 1-0.7 ; 2-1.4 ; 3-2.1 ; 4-2.8$.
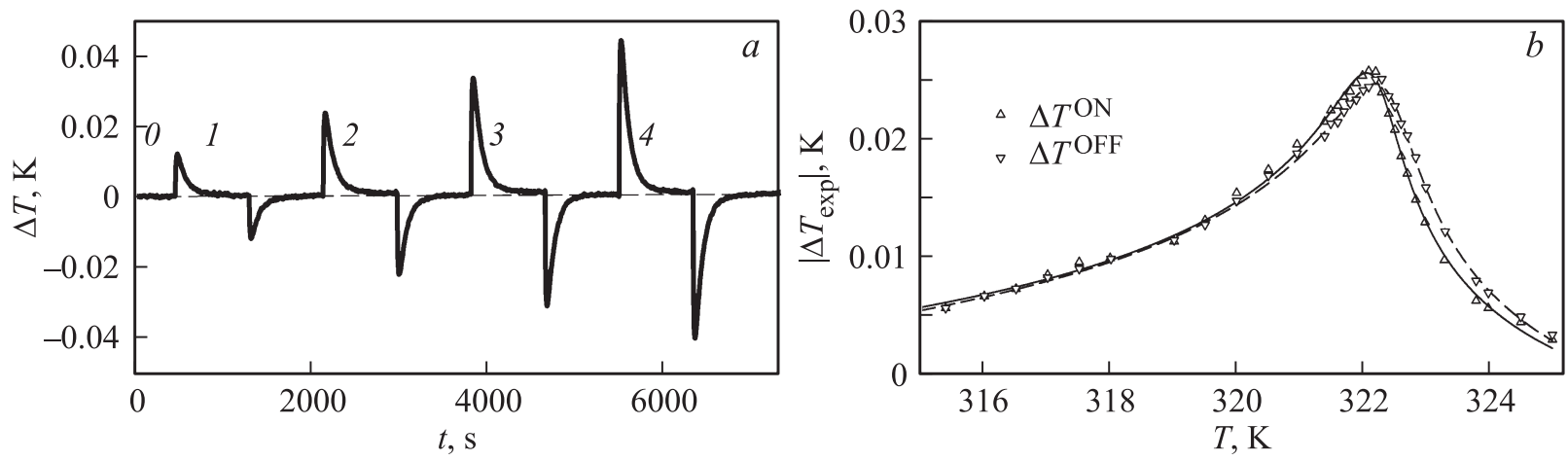

Рис. 5. ЕСЕ в кристалле TGS в квазиизотермических условиях при $T_{\text {bot }}=322.3 \mathrm{~K}$ : результаты измерений $T_{\text {exp }}$ с поэтапным увеличением $E(a)$; температурные зависимости $\left|\Delta T_{\exp }\right|$ вблизи фазового перехода при $E=1.4 \mathrm{kV} \cdot \mathrm{cm}^{-1}(b)$. Номера кривых соответствуют напряженностям $E\left(\mathrm{kV} \cdot \mathrm{cm}^{-1}\right): 0-0.0 ; 1-0.7 ; 2-1.4 ; 3-2.1 ; 4-2.8$.
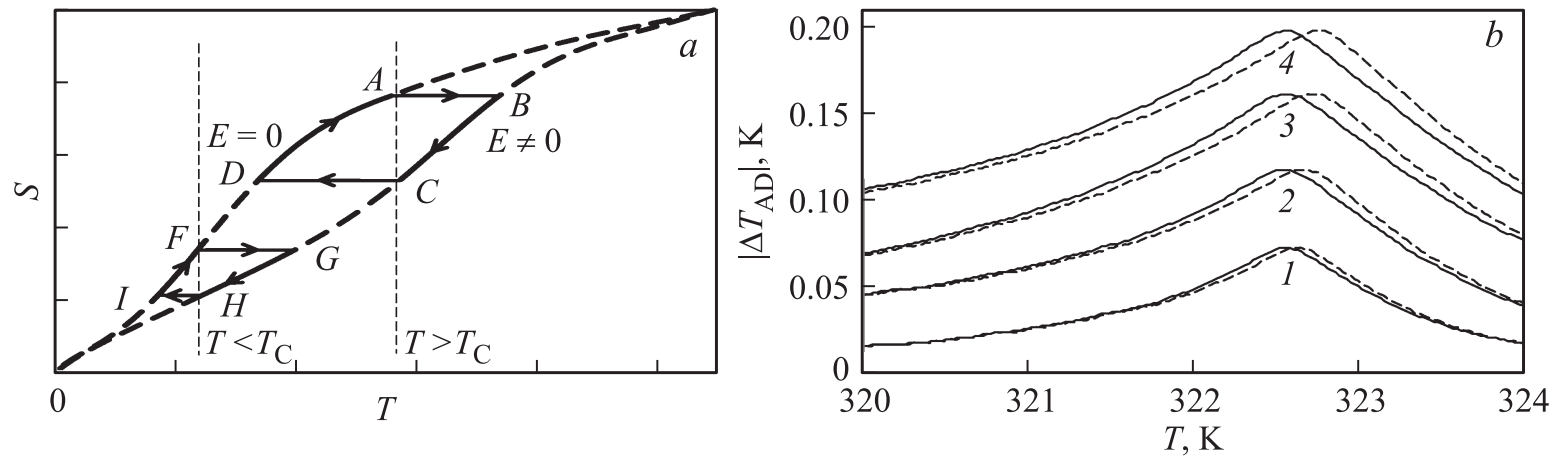

Рис. 6. Циклы в условной диаграмме $S-T$, состоящие из процессов включения/выключения $E$ в условиях $S=$ соnst и релаксации температуры при $E=$ const: $\mathrm{ABCD}-T>T_{C}$ и FGHI $-T<T_{C}(a)$. Температурные зависимости $\left|\Delta T_{\mathrm{AD}}\right|^{\mathrm{ON}}$ (сплошные линии) и $\left|\Delta T_{\mathrm{AD}}\right|^{\mathrm{OFF}}$ (штриховые линии), определенные из диаграммы $S-T$ для TGS $(b)$. Номера кривых соответствуют напряженностям $E\left(\mathrm{kV} \cdot \mathrm{cm}^{-1}\right): 0-0.0 ; 1-0.7 ; 2-1.4 ; 3-2.1 ; 4-2.8$.

стабилизированным) и верхним (термодинамически свободным) торцами ЕС-элемента. Если величины $\left|\Delta T_{\exp }\right|^{\mathrm{ON}}$ и $\left|\Delta T_{\exp }\right|^{\text {OFF }}$ одинаковы, то эквивалентны и количества тепла, переносимые соответственно в направлениях $T_{\text {top }} \rightarrow T_{\text {bot }}$ и $T_{\text {bot }} \rightarrow T_{\text {top. }}$ В этом случае результирующий эффект охлаждения образца отсутствует. Однако экспериментальные данные (рис. 5,b) свидетельствуют, что величины $\left|\Delta T_{\text {exp }}\right|^{\mathrm{ON}}$ и $\left|\Delta T_{\text {exp }}\right|^{\mathrm{OFF}}$ не равны. Следует отметить, что в данном случае коррекция $\left|\Delta T_{\exp }\right|$ в рамках (3) не проводилась, так как трудно было надежно оценить величину теплоемкости $C_{f}$, которая, судя по соотношению между $\left|\Delta T_{\exp }\right|$ (рис. $\left.5, b\right)$ и $\left|\Delta T_{\mathrm{AD}}\right|$ (рис. $\left.4, b\right)$, в несколько раз больше, чем $C_{\mathrm{smp}}$ в „релаксационном“ методе измерений в квазиизотермических условиях. Наи- 

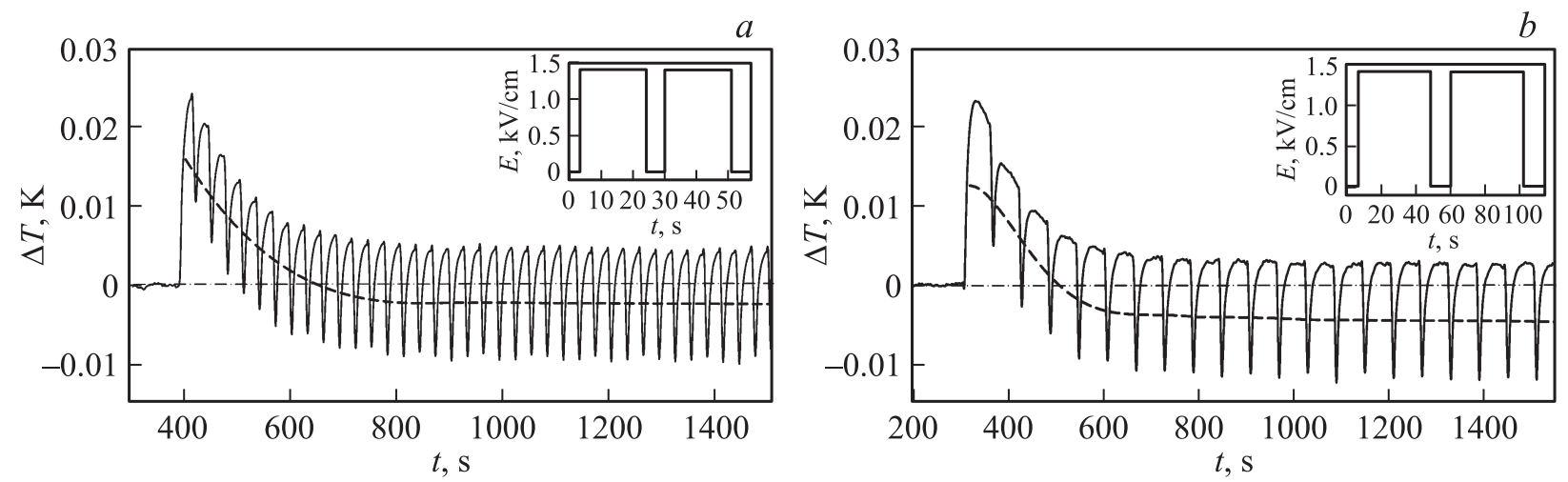

Рис. 7. Зависимость от времени разности температур $\Delta T=T_{\text {top }}-T_{\text {bot }}$ на ЕС-элементе в периодическом электрическом поле $E=1.4 \mathrm{kV} \cdot \mathrm{cm}^{-1}$ при $322.35 \mathrm{~K}: f=0.036 \mathrm{~Hz}(a), f=0.018 \mathrm{~Hz}(b)$. Сплошные линии соответствуют разности между средней величиной $T_{\text {top }}$ и постоянной $T_{\text {bot. }}$. На вставках показан профиль электрического поля.

более важным моментом является то, что соотношения между интенсивными ЕСЕ в рассматриваемом случае (рис. 5,b) были такими же как и в экспериментах, выполненных в адиабатических условиях с $d T / d t<0$ (рис. 4), а именно: при $T<T_{C}\left|\Delta T_{\exp }\right|^{\mathrm{ON}}>\left|\Delta T_{\exp }\right|^{\mathrm{OFF}}$,

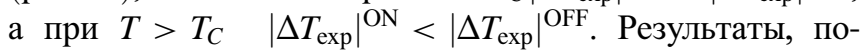
лученные в обсуждаемых релаксационных измерениях, могут быть объяснены с помощью диаграммы $S-T$, где представлено поведение полной энтропии гипотетического ЕС-элемента в окрестностях фазового перехода при $E=0$ и $E \neq 0$ (рис. 6,a).

Циклы $\mathrm{ABCD}\left(T>T_{C}\right)$ и FGHI $\left(T<T_{C}\right)$, состоящие из двух адиабатных и двух изополевых процессов, наглядно демонстрируют наблюдаемые в экспериментах соотношения между $\Delta T_{\mathrm{AD}}^{\mathrm{ON}}$ и $\Delta T_{\mathrm{AD}}^{\mathrm{OFF}}$.

В рамках релаксационного подхода можно проанализировать и данные об ЕСЕ, полученные в исследованиях теплоемкости $C(T, E)$ (рис. 2). В этом случае также наблюдается несовпадение величин $\Delta T_{\mathrm{AD}}^{\mathrm{ON}}$ и $\Delta T_{\mathrm{AD}}^{\mathrm{OFF}}$ выше и ниже $T_{C}$ (рис. $\left.6, b\right)$. Разности $\left|\Delta T_{\mathrm{AD}}\right|^{\mathrm{OFF}}-\left|\Delta T_{\mathrm{AD}}\right|^{\mathrm{ON}}$, полученные в трех независимых экспериментах в параэлектрической фазе при $T=323.6 \mathrm{~K}$, оказались близки между собой: $3.5 \cdot 10^{-3} \mathrm{~K}$ (рис. $4, b$ ), $2.7 \cdot 10^{-3} \mathrm{~K}$ (рис. $5, b)$ и $3 \cdot 10^{-3} \mathrm{~K}$ (рис. $6, b$ ).

Совпадение данных, полученных при разных условиях сопряжения ЕСЕ-элемента с окружающей средой, безусловно, свидетельствует об их высокой надежности.

3.4. Исследование ECE в периодическом электрическом поле. Данные, представленные на рис. 5, можно характеризовать как полученные в периодически меняющемся электрическом поле очень низкой частоты $\sim 6 \cdot 10^{-4} \mathrm{~Hz}$ с амплитудой $E$, увеличивающейся в начале каждого периода. На следующем этапе исследовано влияние частоты $E$ на величину и характер поведения $T_{\text {top }}$ образца при $T_{\text {bot }}=$ const. В отличие от аналогичных измерений, выполненных нами ранее в условиях низкого вакуума $\left(\sim 10^{-2} \mathrm{~mm} \mathrm{Hg}\right)$ [17], рассматриваемые эксперименты в адиабатическом калори- метре при давлении $\sim 10^{-5} \mathrm{~mm} \mathrm{Hg}$ и частотах $0.036 \mathrm{~Hz}$ и $0.018 \mathrm{~Hz}$ удалось провести лишь при $E \leq 1.4 \mathrm{kV} \cdot \mathrm{cm}^{-1}$.

В соответствии с результатами [17] был выбран прямоугольный профиль сигнала (рис. 7) с коэффициентом заполнения 85\%. Исследования ЕСЕ в таких электрических условиях показали, что стационарное состояние системы достигалось после 10-20 импульсов. В результате средняя разность температур $\left(\Delta T^{\mathrm{ON}}+\Delta T^{\mathrm{OFF}}\right) / 2$ между свободным торцом и термостабилизированным основанием ЕС-элемента становилась отрицательной, совершая колебания в пределах $\pm(7-8) \cdot 10^{-3} \mathrm{~K}$ (рис. 7, $\left.a, b\right)$. Частота следования электрических импульсов достаточно сильно влияла на степень охлаждения ЕС-элемента. Так, при $f=0.036 \mathrm{~Hz}$ максимальная средняя разность температур составляет $\left[\left(\Delta T^{\mathrm{ON}}+\Delta T^{\mathrm{OFF}}\right) / 2\right]_{\max }=0.0027 \mathrm{~K}$, а при $f=0.018 \mathrm{~Hz}$ достигает $0.0050 \mathrm{~K}$. С учетом амплитуды колебаний наинизшая температура свободного торца образца достигает $T_{\text {top }}=T_{\text {bot }}-0.012 \mathrm{~K}$.

На первый взгляд, сравнение с данными, полученными в условиях низкого вакуума $\left(\left[\left(\Delta T^{\mathrm{ON}}+\Delta T^{\mathrm{OFF}}\right) / 2\right]_{\max } \approx\right.$ $\approx-0.02 \mathrm{~K})$ [17], оказывается не в пользу приведенных выше результатов. Однако следует учитывать, что в [17] измерения проводились при более низких частотах и более высоких напряженностях $E$.

Все выше рассмотренные эксперименты по определению ECE в TGS были проведены в условиях больших и равных скоростей включения и выключения электрического поля, которые позволяют говорить об адиабатичности соответствующих процессов. Сравнительно недавно был исследован так называемый „кинетический“ ЕСЕ в мультислойном сегнетоэлектрике $\mathrm{BaTiO}_{3}$ [25]. Эксперименты выполнены в дифференциальном сканирующем калориметре, позволявшем проводить измерения количества тепла, выделенного/поглощенного при наложении/снятии электрического поля в изотермических условиях. Оригинальность этой работы заключалась в том, что авторы использовали существенно отличающиеся скорости изменения поля $d E / d t: 1.4 \mathrm{kV} \cdot(\mathrm{cm} \cdot \mathrm{s})^{-1}$ 


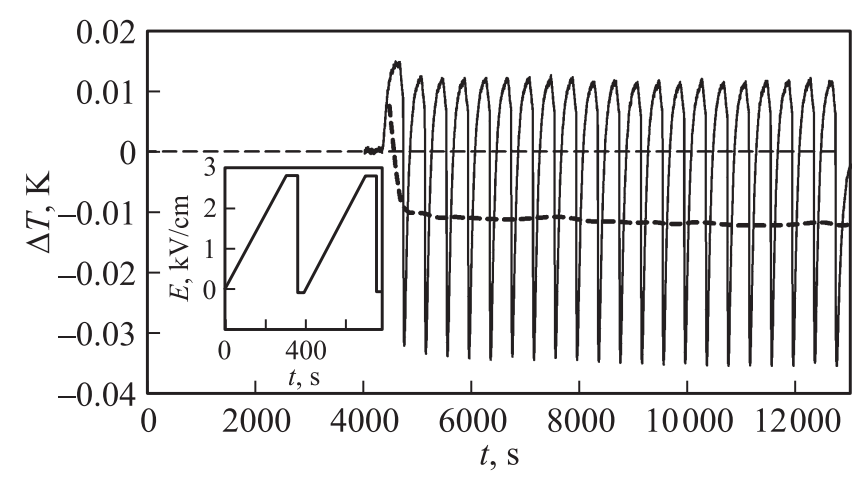

Рис. 8. Зависимость от времени разности температур $\Delta T=T_{\text {top }}-T_{\text {bot }}$ при $322.545 \mathrm{~K}$ в периодическом электрическом поле $E=2.8 \mathrm{kV} \cdot \mathrm{cm}^{-1}, f=0.0025 \mathrm{~Hz}(a)$. Сплошная линия разность между средней величиной $T_{\text {top }}$ и постоянной $T_{\text {bot. }}$. На вставке показан профиль электрического поля.

в процессе $0 \rightarrow E_{\max }$ и $176 \mathrm{kV} \cdot(\mathrm{cm} \cdot \mathrm{s})^{-1}$ при $E_{\max } \rightarrow 0$. Это приводило к тому, что только во втором случае за счет адиабатического (быстрого) снятия поля наблюдался значительный эндотермический эффект в узком интервале времени.

По этой же методике нами выполнены исследования влияния дисбаланса скоростей в процессах $0 \rightarrow E_{\max }$ и $E_{\max } \rightarrow 0$ на ECE в TGS. В результате установлены оптимальные форма сигнала и частота электрического поля, позволяющие получить более значительные параметры охлаждения (рис. 8).

Для электрического поля $E=2.8 \mathrm{kV} \cdot \mathrm{cm}^{-1}$ и $f=$ $=0.0025 \mathrm{~Hz}$ при степени заполнения 93\% наибольшая средняя разность температур достигала более существенной величины $\left[\left(\Delta T^{\mathrm{ON}}+\Delta T^{\mathrm{OFF}}\right) / 2\right]_{\max }=-0.012 \mathrm{~K}$ по сравнению с измерениями с прямоугольным профилем $E$. При этом наинизшая температура свободного торца ЕС-элемента была ниже $T_{\text {bot }}$ на $\sim 0.035 \mathrm{~K}$.

\section{4. Заключение}

Методом адиабатического калориметра выполнены исследования интенсивного ЕСЕ $\Delta T_{\mathrm{AD}}$ в области сегнетоэлектрического фазового перехода в кристалле TGS. Изучены некоторые аспекты влияния равновесных и неравновесных термодинамических условий (адиабатических, квазиизотермических) сопряжения ЕС-элемента с окружающей средой и параметров электрического поля на величину и степень обратимости $\Delta T_{\mathrm{AD}}$ и установлены следующие важные моменты.

1. Результаты прямых измерений ЕCE в адиабатических условиях в режимах включения и выключения поля при разных температурах, соответственно $T$ и $T+\Delta T_{\mathrm{AD}}$, характеризуются высокой степенью воспроизводимости и обратимости величин $\Delta T_{\mathrm{AD}}^{\mathrm{ON}}$ и $\Delta T_{\mathrm{AD}}^{\mathrm{OFF}}$. Несовпадение значений $\Delta T_{\mathrm{AD}}(\sim 20 \%)$, непосредственно измеренных и определенных из диаграммы $S-T$, построенной на основании полученных в работе данных о влиянии электрического поля на теплоемкость TGS, не является удивительным в соответствии с анализом, выполненным в [21]. Обнаруженная в экспериментах проводимость кристалла TGS является причиной выделения джоулева тепла на сопротивлении образца при $E \neq 0$ и необратимого вклада в изменение его температуры.

2. Выполнены исследования влияния на интенсивный ECE неравновесности процесса $E=$ const, когда температура ЕС-элемента понижается $\left(T+\Delta T_{\mathrm{AD}}\right) \rightarrow T$, то есть включение и выключение электрического поля происходит при одной и той же температуpe (рис. 4, $a, 5, a, 6, a)$. Удовлетворительно согласующиеся данные трех независимых экспериментов свидетельствуют о несовпадении величин $\Delta T_{\mathrm{AD}}^{\mathrm{ON}}$ и $\Delta T_{\mathrm{AD}}^{\mathrm{OFF}}$ и их разном соотношении в сегнетоэлектрической $\left|\Delta T_{\mathrm{AD}}\right|^{\mathrm{ON}}(T, E)>\left|\Delta T_{\mathrm{AD}}\right|^{\mathrm{OFF}}(T, E)$ и параэлектрической $\left|\Delta T_{\mathrm{AD}}\right|^{\mathrm{ON}}(T, E)<\left|\Delta T_{\mathrm{AD}}\right|^{\mathrm{OFF}}(T, E)$ фазах (рис. $4, b$, $5, b, 6, b)$.

3. Исследования ЕСЕ в периодическом электрическом поле в квазиизотермических условиях выявили существенное влияние профиля импульса и частоты на разность температур термостатированного $T_{\text {bot }}$ и „свободного“ $T_{\text {top }}$ торцов ЕС-элемента. Уменьшение частоты поля приводит к увеличению $T_{\mathrm{bot}}-T_{\mathrm{top}}$, как это наблюдалось и в условиях значительно более низкого вакуума [17]. Однако такая зависимость глубины охлаждения от частоты является противоположной ожидавшейся в расчетных работах $[15,16]$. Можно предполагать, что это различие, вероятнее всего, связано с процессами переполяризации и перестройки доменной структуры кристалла TGS. Вполне вероятно, что с ростом частоты кристалл не успевает поляризоваться/деполяризоваться и в результате величина ЕСЕ, пропорциональная $(\partial P / \partial T)$, существенно уменьшается.

Таким образом, очевидно, что приложение периодического электрического поля к ЕС-элементу, находящемуся в неравновесных термодинамических условиях, связанных с фиксацией температуры одного из его торцов, позволяет реализовать температурный градиент вдоль ЕС-элемента. Установленные экспериментально небольшие разности $T_{\text {top }}-T_{\text {bot }}$ не позволяют говорить о значительных тепловых потоках между свободным и термостатированным торцами EC-элемента, по крайней мере, в случае сегнетоэлектрика TGS. Однако, учитывая, что все данные получены в полях низкой напряженности, безусловно, представляют интерес и являются перспективными дальнейшие исследования на материалах, обладающих более значительным ЕСЕ, и в условиях более высоких полей. Наиболее привлекательным аспектом подобного рода работ является гипотетическая возможность реализации системы охлаждения, не требующей тепловых ключей.

C учетом сказанного выше, на наш взгляд, представляет интерес модификация модели охлаждающей линии, предложенной в $[15,16]$, путем учета эффектов переполяризации и проводимости. 


\section{Список литературы}

[1] M. Tishin, Y. Spichkin: The Magnetocaloric Effect and its Application. Bristol, Philadelphia (2003). 475 p.

[2] K.A. Gschneidner Jr., V.K. Pecharsky, A.O. Tsokol. Rep. Prog. Phys. 68, 1479 (2005).

[3] J.F. Scott. Ann. Rev. Mater. Res. 41, 229 (2011).

[4] M. Valant. Prog. Mater. Sci. 57, 980 (2012).

[5] A. Smith, C.R.H. Bahl, R. Bjørk, K. Engelbrecht, K.K. Nielsen, N. Pryds. Adv. Energy Mater. 2, 1288 (2012).

[6] S. Crossley, N.D. Mathur, X. Moya. AIP Advances 7, 067153 (2015).

[7] X. Moya, S. Kar-Narayan, N.D. Mathur. Nature Mater. 13, 439 (2014).

[8] И.Н. Флёров, Е.А. Михалёва, М.В. Горев, А.В. Карташев. ФTT 57, 421 (2015).

[9] B. Asbani, J.-L. Dellis, A. Lahmar, M. Courty, M. Amjoud, Y. Gagou, K. Djellab, D. Mezzane, Z. Kutnjak, M.El. Marssi. Appl. Phys. Lett. 106, 042902 (2015).

[10] H. Liu, X. Yang. AIP Advances 5, 117134 (2015).

[11] H.Y. Lee, K.H. Cho, H.-D. Nam. Ferroelectrics 334, 165 (2006).

[12] A.S. Mischenko, Q. Zhang, J.F. Scott, R.W. Whatmore, N.D. Mathur. Science 311, 1270 (2006).

[13] A.S. Mischenko, Q. Zhang, J.F. Scott, R.W. Whatmore, N.D. Mathur. Appl. Phys. Lett. 89, 242912 (2006).

[14] А.С. Старков, С.Ф. Карманенко, О.В. Пахомов, А.В. Еськов, Д. Семикин, J. Hagberg. ФТТ 51, 1422 (2009).

[15] I. Starkov, A. Starkov. Ferroelectrics 480, 102 (2015).

[16] А.В. Еськов, С.Ф. Карманенко, О.В. Пахомов, А.С. Старков. ФТТ 51, 1483 (2009).

[17] V.S. Bondarev, E.A. Mikhaleva, M.V. Gorev, I.N. Flerov. Phys. Status Solidi B 253, 2073 (2016).

[18] А.В. Карташев, И.Н. Флёров, Н.В. Волков, К.А. Саблина. ФTT 50, 2027 (2008).

[19] С.А. Тараскин, Б.А. Струков, В.А. Мелешина. ФТТ 12 , 1386 (1970).

[20] Е.Ф. Дудник, В.М. Дуда, А.И. Кушнарев. ФТТ 42, 139 (2000).

[21] Y. Liu, J.F. Scott, B. Dkhil. Appl. Phys. Rev. 3, 031102 (2016).

[22] Л.Д. Ландау, Е.М. Лифшиц. Статистическая физика. М., Наука (1964). 567 c.

[23] L. Tocado, E. Palacios, R. Burriel. JMMM 290-291, 719 (2005).

[24] Б.А. Струков. Кристаллография 11, 892 (1966).

[25] Y. Bai, G.-P. Zheng, S.-Q. Shi. J Appl Phys. 108, 104102 (2010). 